\title{
Intangibles para el proceso de diseño en el marco de la sociedad del conocimiento
}

\author{
Matovelle-Villamar, Rutha \& Lecuona-López, Manuel Ramón ${ }^{\text {b }}$ \\ ${ }^{\mathrm{a}} \mathrm{PhD}$ candidate - Universitat politècnica de València, Spain \& Escuela Superior Politècnica del Litoral, Ecuador. \\ rmatovel@espol.edu.ec \\ ${ }^{\mathrm{b}} \mathrm{PhD}$ Full time Professor - Universitat politècnica de València, Spain. mlecuona@upv.es
}

\begin{abstract}
Resumen
Hoy vivimos en la llamada "sociedad del conocimiento", la cual ha generado grandes trasformaciones en el quehacer empresarial. Las empresas han pasado de un sistema productivo, basado en factores materiales, a un sistema económico constituido por factores cognitivos, creativos e informativos que contribuyen cada vez más a la riqueza de las empresas. En este contexto, el objetivo del artículo es identificar las nuevas formas de conocimiento que transforman, especificamente, la dimensión empresarial y su vinculación con los componentes intangibles, cuyo tratamiento se convierte en insumos del proceso de diseño. Para su desarrollo, se optó por una investigación descriptiva de la literatura existente, que permita crear un estado del arte para conocer qué aspectos se han investigado y cuales permanecen ignorados. En este sentido, se pudo evidenciar que el mayor reto para las empresas es la gestión apropiada del conocimiento, con el que se alcance un posicionamiento competitivo y asegure su supervivencia. La disciplina de diseño desempeña un papel estrategico en la consecución de este objetivo, pues, bajo una visión sistémica, realiza la función transformadora de buscar nuevas estrategias, que le permitan identificar y mejorar la gestión de los intangibles empresariales, con la clara intención de innovar y optimizar el diseño de lo tangible.
\end{abstract}

Palabras clave: sociedad del conocimiento; intangibles empresariales; proceso de diseño; diseño estrategico, innovación.

\begin{abstract}
Nowadays we live in what is called the "knowledge society" which has generated great transformations in business activity. Companies have gone from a material based production system, to a knowledge based productive system, where creative and information related factors are contributing increasingly to the wealth building of businesses. In this context, the objective of this article is to identify new forms of knowledge that can transform this business dimension and its relationship to the intangible components, such treatment becomes an important input in the design process. For its development, we chose a descriptive research of existing literature, allowing to create a state of the art in order to know what aspects have been investigated and which remain unknown. It became evident that the biggest challenge for companies is proper knowledge
\end{abstract}


management, thereby achieve a competitive position and ensure its survival. The design discipline plays a starring role in achieving this objective, therefore, under a systemic, performs the transformative role of new strategies, allowing him to identify and improve management of business intangibles, with the clear intention to innovate and optimize the design of the tangible.

Keywords: knowledge society; business intangibles; design process; innovation.

\section{Introducción}

El paso del tiempo, ha marcado históricamente transformaciones que han afectado a todo tipo de empresas, sean estas pequeñas, medianas o grandes. Los historiadores han agrupados estas transformaciones en tres sociedades: la Agrícola, la Industrial y la del Conocimiento, también identificada con los términos: "Sociedad Post-industrial"(Bell,1991), "Sociedad de la Información", "Sociedad Red" o "Los Flujos" (Castells, 1999). Por lo que resulta necesario realizar una rápida mirada del pasado, para visualizar el hoy.

La sociedad agrícola. Es la más larga de la historia, fue una etapa dominante a través de la cual el ser humano lograba su subsistencia y desarrollo, se cuenta sus inicios desde que el hombre está en la tierra, hasta finales del siglo XIX. (Giner de la Fuente \& Gil Estallo, 2014).

La Sociedad industrial.- Surgió a mediados del siglo XVIII como consecuencia de la revolución Industrial iniciada en Inglaterra, se extendió por Europa y en o corto plazo al resto del mundo. La productividad fue el valor más deseado, cada individuo era considerado un engranaje del sistema que producía la mayor cantidad de mercancía posible en el menor tiempo posible. Esto represento grande movimientos migratorios, del campo a la ciudad que es dónde se concentraron las fábricas, el sociólogo Marx enfoca el trabajo del obrero como "El trabajo externo, el trabajo en que el hombre se enajena, es un trabajo de auto sacrificio, de ascetismo" (Marx, 1844).

La sociedad de conocimiento, tiene sus orígenes en los años noventa cuando se analizaron los cambios en las sociedades industriales, surgió el término sociedad post-industrial, introducido por el sociólogo Daniel Bell( 1973; 2001), "este tipo de sociedad está orientado hacia el progreso tecnológico y la evaluación de la tecnología y se caracteriza por la creación de una nueva tecnología intelectual como base de los procesos de decisión" (Bell, 1991, pág. 53). Esta es la sociedad de la globalización y las Tics, y en ella nos encontramos hoy.

El sociólogo Manuel Castells, centra sus estudios en analizar las afectaciones de esta nueva sociedad desde varias ópticas: los movimientos sociales urbanos, el surgimiento de la sociedad en red, y el rol de las ciudades en la economía basada en información. Sostiene que "debido a que la materialidad de nuestra existencia está hecha de flujos y/o de resistencias a estos flujos basados en la comunidad, la representación de los valores e intereses en nuestras sociedades ya no se estructura sobre la base del trabajo, sino que se expresa en términos de un mensaje simbólico, los flujos no son sólo un elemento de la organización social, son la expresión de los procesos que dominan nuestra vida económica, política y simbólica" (Castells, 1999). Estas afirmaciones, permiten visualizar claramente que la sociedad se transforma a través de sus diversas dimensiones: cultural, económico/empresarial, la planificación espacial y la tecnológica, proporcionando modelos globales con múltiples interconexiones. El presente artículo tiene como contexto específicamente las transformaciones ocurridas en la dimensión empresarial, a lo que surge los cuestionamientos : ¿qué cambios experimentan las empresas?, ¿qué relación tiene estos 
cambios con la presencia o generación de activos intangibles? y ¿en que contribuye la inminente presencia de los intangibles, con el trabajo de los profesionales del área de diseño?

La presente investigación pretende dar contestación a cada una de las preguntas planteadas, para lo cual su objetivo es identificar las nuevas formas de conocimiento que transforman, específicamente, la dimensión empresarial y su vinculación con los componentes intangibles, cuyo tratamiento se convierte en insumos del proceso de diseño.

\section{Antecedentes teóricos.}

\subsection{La sociedad del conocimiento y su impacto en las empresas.}

Drucker en su libro "La era de la discontinuidad" da origen al término "la sociedad del conocimiento", es reconocido como padre y mentor conjunto con Fritz Machlup (Drucker, 1969). El ganador del premio Nobel de Economía en 1987, Robert Solow, fue uno de los primeros en señalar la importancia del conocimiento en el crecimiento económico, expresando que este es una función de capital, trabajo y conocimiento, sus estudios sobre esta temática, fueron la base para otorgarle el Premio Nobel. Sus estudios incluían análisis numéricos sobre el crecimiento económico de Estados Unidos en la primera mitad del siglo XX, en la que se evidenciaba un residuo inexplicable, que no es el resultado del incremento de los factores de capital y trabajo. La parte no explicada se denominó "residual de Solow", que correspondía a algo más, al progreso técnico, como lo llamó Solow, o simplemente conocimiento. (Corrado, Hulten, \& Sichel, 2004).

Este conocimiento, está transformando radicalmente las economías, los mercados y la estructura de la industria, los productos y servicios, los puestos de trabajo y los mercados laborales (Drucker, 1969). Las antiguas tradiciones a nivel empresarial se han visto gravemente afectadas, Idris Moote menciona que "las empresas están sufriendo una gran turbulencia cultural constante con afectación directa sobre reputación, crecimiento y rentabilidad" (Mootee, 2014, pág. 3). Calleja (2001), señala que "las empresas como organizaciones humanas con objetivos de creación de riqueza y bienestar, están afectadas por el crecimiento de la complejidad y deben establecer mecanismos para tenerla en cuenta y moverse con éxito en un entorno más interdependiente". Por otra parte Morán y Brightman afirman que las empresas deben "renovar continuamente de dirección, estructura y capacidades de la organización para servir a las necesidades siempre cambiantes de los clientes externos e internos" (Moran \& Brigthtman, 2001, pág. $115)$, lo que lleva a pensar que las empresas se enfrentan a una gran incertidumbre.

Estos acontecimientos, han originado numerosas investigaciones a nivel mundial, bajo el enfoque de identificar las causas y posibles soluciones a las transformaciones empresariales. Aguilá y Monguet desarrollaron una metodología para guiar la evolución desde modelos de negocio obsoletos a modelos propios del siglo XXI, ellos exponen un esquema analítico para la reconstruir la oferta del producto y servicio (Aguilá \& Monguet, 2010). Los autores Giner de la Fuente \& Gil Estallo, en cambio se enfocan en "estudiar como las TIC están cambiando de forma radical la división de trabajo dentro de las empresas y con ello enviando a la basura los paradigmas de la gestión de la empresa" (Giner de la Fuente \& Gil Estallo, 2014). Moote, propone una metodología sobre como insertar en la empresa moderna un nuevo conjunto de instrumentos basados en el Design Thinking, que permita una nueva oleada de colaboración, visión y aprendizaje, destinados a mejorar la toma de decisiones (Mootee, 2014). Los autores Anargyros \& Loeb,en su obra ¿Y si ponemos los relojes de nuevo a cero?, ofrecen una visión particular desde el Diseño de las transformaciones empresariales: "repensar el presente desde diferentes puntos de vista ofreciendo estímulos para darse cuenta de futuros posibles, el futuro no existe, pero con el fin de desarrollar hipótesis del futuro, hay que mirar más de cerca la actualidad, la zona cero" (Anargyros \& Loeb, 1998). La mayoría de estos estudios tienen como punto de partida, que la empresa tome conciencia de la situación, a través de la identificación de las fuerzas externas a las que están sometidas actualmente. El presente articulo ha tomado de base las fuerzas externas analizadas por los autores Aguilá y Monguet, a saber:

- Globalización, partiendo de un mercado local o regional, se ha evoluciona a los internacionales y a mundiales, alcanzables gracias a la presencia de la tecnología, la internet rompió todos las barreras físicas y territoriales. Tanto las ventas como, las tareas deben 
realizarse bajo una visión planetaria. Los ciclos económicos están fuertemente caracterizados por la mundialización.

- La sostenibilidad del planeta, la crisis de los recursos naturales, es más urgente que la económica y empeora cada vez más a medida que aumenta la población, y no hay que olvidar el reconocimiento de los derechos de las minorías.

- La innovación tecnológica, no solo la informática y las comunicaciones, la bioingeniería, los nuevos materiales, la nanotecnología están provocando continuamente revoluciones en los mercados.

- Demografía, grandes migraciones procedentes principalmente de países en desarrollo, hacia países desarrollados, generan cambios en la cultura y costumbres occidentales, los cuales poseen en su mayoría bajas tasas de natalidad.

- La saturación de oferta de productos, la eminente necesidad de reformular la oferta de los mercados, considerando una diferenciación que asegure la supervivencia en el mercado.

- La necesidad cambiante de los usuarios, lo que fomenta la co-creación con los clientes, esto generá elementos de confianza mutuos.

\subsection{Los intangibles empresariales.}

La intangibilidad $^{25}$ se manifiesta en las dificultades de medir, tocar y manipular, el término "activos intangibles", es definido por distintos organismos internacionales como la FASB (Financial Accounting Standards Board) en los Estados Unidos o el ASB (Accounting Standards Board) en el Reino Unido, y por la Norma Internacional de Contabilidad NIC $38^{26}$, en forma similar (Cañibano \& Gisbert , 2005) como:“ identificable, de carácter no monetario y sin apariencia física” (NIC 38, 2004 , párrafo 8). La importancia de medir los activos intangibles dentro de las organizaciones modernas radica en que estos representan un recurso crucial para la sostenibilidad de los beneficios económicos en el largo plazo.

Desde la década de 1990 viene creciendo la inquietud sobre la necesidad de analizar y medir los diferentes tipos de activos intangibles para incluirlos en los estados financieros de las empresas. Este ha sido el propósito de numerosas investigaciones, desde variadas perspectivas: la contable, el capital intelectual, medida de desempeño y la valoración financiera. Para citar solo un ejemplo, el artículo "Study on the measurement of intangible assets and associated reporting practices", detalla 23 métodos de medición de activos intangibles. (European Commission, 2003). Por otra parte, la empresa Ocean Tomo con sede en Chicago, Estados Unidos, realizo un estudio que media el peso de los componentes tangibles e intangibles en el valor de mercado, a una muestra de 500 empresas que componen el índice S\&P500. El período de observación fue de 35 años, en él se evidenció un cambio drástico en la proporción de los componentes que soportan el valor de mercado de las empresas, al pasar del 83\% tangible vs. el 17\% intangible en el año 1975, a una inversión de la proporción, con un 20\% tangible vs. un 80\% intangible en 2010 (Torres, 2014).

Otra contribución influyente sobre el crecimiento y valoración de activos intangibles lo dan Carol Corrado, Charles Hulten y Daniel Sichel (en su momento investigadores del Federal Reserve Board y de la University of Maryland), identificada como CHS, la que se ha convertido en la metodología estándar en la literatura económica actual. Esta considera que los gastos que se realizan las empresas en diseño, marketing, formación del personal o mejoras organizativas, son tan importantes como los gastos en I+D (Corrado, Hulten, \& Sichel, 2004). Esta metodología ha sido utilizada por otros centros de investigación, tal como el proyecto INTAN-Invest [www.intan-invest.net], aplicada para calcular la inversión en intangible de un grupo de países europeos. Los autores han continuado con sus investigaciones y sus trabajos propiciaron la creación de organizaciones como Innodrive,

\footnotetext{
${ }^{25}$ Intangible: adj. Que no debe o no puede tocarse. Diccionario de la Lengua Española. Real Academia Española. vigésima primera edición, 1992

${ }^{26}$ El objetivo de esta Norma es prescribir el tratamiento contable de los activos intangibles, especifica los criterios que permiten reconocer un activo intangible y también especifica cómo determinar el importe en libros de los activos intangibles
} 
establecida bajo los auspicios de la Comunidad Europea, quien ha financiado numerosos estudios para entender cabalmente la importancia de los activos intangibles en la sociedad del conocimiento.

La metodología CHS, identifico y clasifico en tres grupos a los activos intangibles, que le permiten realizar su respectiva cuantificación: Información computarizada (software y bases de datos); Propiedad científica y creativa (I\&D, exploración minera, derechos de autor y costos de licencia, desarrollo de productos, diseño y otros gastos de investigación); Competencias económicas (valor de marca, capital humano, estructura organizativa, modelo de negocios) (Corrado, Hulten, \& Sichel, 2004, pág. 40).

\section{Metodología.}

La metodología aplicada en este artículo, fue realizar una revisión descriptiva de la literatura existente sobre la afectación del conocimiento en el quehacer empresarial y su vinculación con los intangibles empresariales. Para la localización de los documentos bibliográficos se utilizaron varias fuentes documentales. La búsqueda se realizó utilizando los descriptores: sociedad del conocimiento, intangibles empresariales y proceso de diseño. Otro criterio de validación fue considerar publicaciones en español o inglés, en las que se hayan obtenidos resultados debidamente respaldados, con información obtenida de un proceso meticuloso de investigación.

\section{Resultados.}

Luego del análisis de la bibliografía recogida, sobre el nivel de afectación que realiza la presente sociedad del conocimiento, a la dimensión empresarial. Se evidencio que esta, trastorna principalmente el modelo de negocio de la empresa, lo que conlleva a una redefinición de su estructura organizacional y ocupacional, y por ende un cambio radical en sus procesos. Entre las fuerzas externas que originan el cambio en el modelo de negocio se encuentran: la globalización, la innovación tecnológica, la sostenibilidad del planeta, la demografía, saturación de oferta de productos y servicios y la necesidad cambiante de los usuarios.

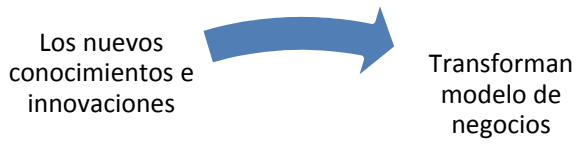

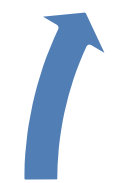

Cambian el modelo ocupacional modelo de
negocios

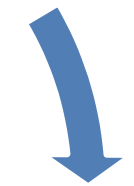

Cambian modelo organizacional

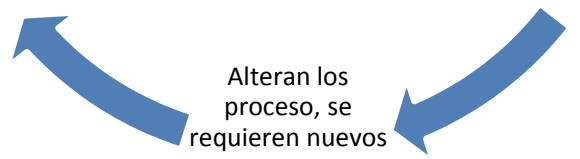

Fig 1. El ciclo de afectación en la dimensión empresarial, causada por la sociedad del conocimiento. Fuente : Los autores. 
Estos elementos externos impactan sobre la empresa, obligandola a reemplantearse su oferta desde una visión interna, esto ha dado origen al crecimiento y valoración de los activos intangibles, Drucker considerado el mayor filósofo de la administración del siglo XXI, menciona que "la empresa debe enfocarse específicamente en la complejidad de los activos intangibles, pues es aquí donde está su mayor crecimiento, para lograr una ventaja competitiva sostenible, y en esencia un conocimiento productivo" (Drucker, El management del siglo XXI, 2000). La identificación de cuáles son los intangibles empresariales, resultantes de la gestión del conocimiento ha tenido muchas opiniones, también numerosas metodologías que permiten su medición e incorporación en los estados financieros (explicación detallada en antecedentes téoricos). El presente articulo ha tomado de base la metodologia CHS, la que clasifica en tres grupos a los activos intangibles: Información computarizada, Propiedad científica y creativa y Competencias económicas (Corrado, Hulten, \& Sichel, 2004, pág. 40). Para efectos de medición, esta se descompone en 9 indicadores.

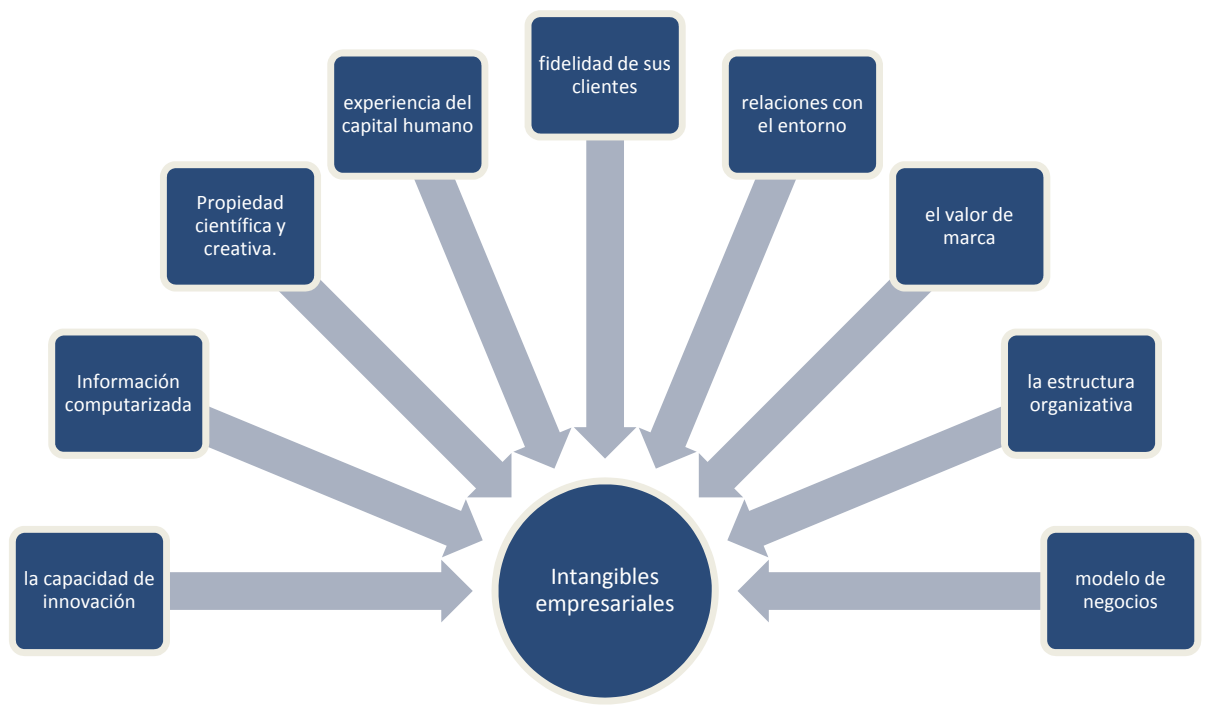

Fig. 2. Los intangibles empresariales. Fuente : Los autores.

Potenciar estos intangibles es la nueva estrategia empresarial, a la que deben estar alineados todos los procesos, incluyendo el de Diseño. El Diseño desde la optica de proceso, esta presente desde las fases de detección de portunidades y analisis de las necesidades del Mercado, hasta las fases de comercialización del producto. En este intervalo confluyen campos de actividad tan diversos como marketing, ingeniería, sistemas de información, etc. Este proceso desde una óptica sistémica, tal como lo describe el Modelo de Diseño Concurrente de Hernandis, específica que "las Variables de Entrada (VE) dadas desde el sistema exterior influencian los aspectos a considerar en el diseño, aportando valores cualitativos o cuantitativos que actúan sobre el Sistema" (Hernandis, 2010). Sería necesario entonces que estas VE, contemplen las variables internas de los intangibles empresariales. Esto nos coloca en un nuevo escenario, la inminente necesidad de observar y estudiar desde la perspectiva de Sistemas, los intangibles empresariales en el proceso de diseño, tal como lo menciona Evers "mientras los conocimientos aumentan con gran rapidez, el saber de lo que no sabemos aumenta con velocidad aún más vertiginosa". (H. D. Evers 2000. p. 8). En este contexto, se ha encontrado algunos esfuerzos desarrollados por investigadores que buscan optimizar el proceso de diseño como función transformadora, captando la intangibilidad empresarial: Donald Norman y Roberto Verganti; Alessandro Deserti y Francesca Rizzo; Francesco Zurlo y Cabirio Cautela y David Aaker. 


\subsection{Capacidad de Innovación}

Donald A. Norman ${ }^{27}$, uno de los originadores del diseño centrado en el usuario -HCD (por sus siglas en inglés human-centered design)" y Roberto Verganti un estudioso de la gestión de la innovación tecnológica. Utilizan de base a las teorías establecidas en el campo de la innovación y la investigación científica / tecnológica, que distinguen la innovación radical e incremental, básica y la investigación aplicada, analizan muchos casos en las que el diseño impulsa a la innovación, llegando a una conclusión. "Una innovación radical surge sin ningún tipo de investigación de diseño o análisis formal de las necesidades de una sociedad o sector en particular, surgen impulsado por los cambios tecnológicos" (Norman \& Verganti, 2014, pág. 84), citan un ejemplo reciente tales como Facebook, twitter y las redes sociales, surgieron simplemente porque sus inventores pensaron que eran cosas interesantes para probar. Generalmente una innovación radical genera una disrupción. Norman no pudo encontrar ningún ejemplo de innovación radical que resultó del proceso diseño centrado en el usuario. Una vez que la innovación radical se había desarrollado, sin embargo, el diseño centrado en el usuario (HCD) fue invaluable como una manera de mejorar el producto. La mejora de sus ejemplos es que Google, Facebook y Twitter se han modificado a sí mismos desde su introducción inicial (Norman \& Verganti, 2014, pág. 79).

\subsection{Estructura Organizativa}

Alessandro Deserti ${ }^{28}$, investigador en el área de gestión del diseño, e innovación a través del diseño. Francesca Rizzo ${ }^{29}$, investigador en el área de diseño de servicios y diseño participativo, realizan una investigación, que se centra en explorar la idea de que el diseño de nuevos productos podría traer cambios inesperados en la cultura de una empresa, ya que su desarrollo puede generar contradicciones entre la cultura actual y la que se necesita para poner en práctica la innovación. Los autores proponen una perspectiva de abajo hacia arriba en el cambio organizacional, vinculándola a la observación de casos reales y la situacionalidad de la práctica del diseño y la cultura como un valor posible, en contraste con la idea de modelos y técnicas que supuestamente pueden ser aplicado en cualquier contexto y situación. Por esta razón, los autores critican el cambio de arriba hacia abajo desde el punto de vista de gestión y pensamiento de diseño; como inadecuados para hacer frente a los cambios e innovación. Esta investigación también realiza un aportación sobre el tema que el producto o servicio, a ser desarrollado no debe únicamente considerar en su fase inicial las necesidades externas de los clientes, sino este debe poder transmitir la cultura de la empresa (Deserti \& Rizzo, 2014, pág. 37).

\subsection{Técnología}

Francesco Zurlo ${ }^{30}$ y Cabirio Cautela ${ }^{31}$, proponen que el diseñador debe saber escuchar las narrativas empresariales en el rol de intérprete-lector, para luego poner en acción una contra-narración animada por la forma y la lógica del proceso creativo, cuyos resultados se expresan como conceptos y prototipos, proceso que cambiaría de acuerdo a los diversos contextos productivos. Dan una amplia explicación

\footnotetext{
${ }^{27}$ Donald Norman cofundó el Nielsen Norman Group, consultoría dedicada a la usabilidad, profesor emérito de la Universidad de California, San Diego (UCSD), tanto en Ciencia Cognitiva y Psicología. Asesor empresarial y organizaciones como el Instituto de Diseño de Chicago y ex Vicepresidente de Tecnología Avanzada de Apple. Sus últimos estudios están enfocados en cómo se conjugan las emociones y diseño en el uso de los productos, tema desarrollado en su libro "emotional design".

${ }^{28}$ Alessandro Deserti ,profesor del Politécnico de Milano, departamento de Diseño, Investigó las nuevas funciones de diseño dentro de las empresas, instituciones y entornos sociales, ha publicado libros, ensayos, artículos en revistas y actas de congresos internacionales.

${ }^{29}$ Francesca Rizzo, profesora adjunto en la Universidad de Bolonia, Departamento de Arquitectura, imparte clases de Diseño Industrial. Ella trabajó en diferentes proyectos de investigación europeos y nacionales en el campo de Diseño de Interacción y Diseño del Servicio. Es autor de numerosos artículos publicados en las actas de congresos ( DPPI , HCI , IASDR , PD ; DRS) y revistas ( Codesign, Tecnología y Cognición, Comunicación de la ACM ).

${ }^{30}$ Francesco Zurlo, catedrático en el Politécnico de Milán, Director del Master en Diseño Estratégico .Ha sido el director general de Polidesign - 2004-2008. Fue galardonado con el 'Golden Compass 2001 (el premio de diseño más prestigiosos de Italia). Ha publicado más de 40 artículos, entre ellos seis artículos en revistas internacionales sobre cuestiones de diseño e innovación y publicado seis libros.

${ }^{31}$ Cabirio Cautela, profesor asistente del Politécnico de Milán, Phd en Business Management. Ha sido visitante invitado en Stanford University - CDR (Center for Design Research) en el 2012. Sus Investigaciones giran sobre el rol estratégico del Diseño, el manejo de procesos de diseño. Es co-director del master en Strategic Design.
} 
teórica documentada acerca de lo significa "las narrativas ${ }^{32}$ del negocio“ creadas por las empresas, según (Czarniawska, 1997), que demuestran que pueden ser usadas en sus relaciones con los diseñadores. En su metodología propuesta establecen que existen dos variantes: el mercado y la tecnología, con sus respectivas opciones de elección, viejo y lo nuevo, crea cuatro campos narrativos diferentes, como se muestra en la Figura 3: la narrativa de explotación, la tecno-narrativa, el relato centrado en el usuario y el exploratorio. Los autores quieren llegar a determinar y principalmente a dotar a los diseñadores, el camino a seguir dependiendo de lo que trasmita la narrativa empresarial, viéndole desde una óptica sistémica, se podría indicar que identifica claramente variables, que servirán para alimentar la primera etapa del proceso de diseño (ver tabla 1) (Zurlo \& Cautela, 2014).

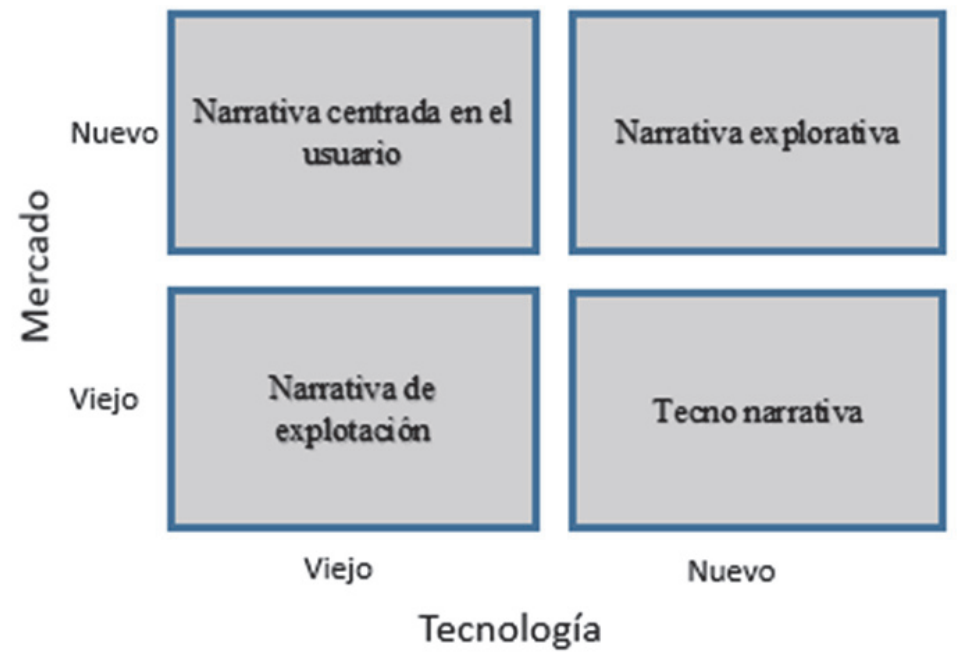

Fig. 3 Las narrativas empresariales. Fuente: DesignIssues: Volume 30, Number 1 Winter (2014).

Tabla 1. Las Narrativas empresariales versus los factores que promueven el proceso de diseño.

\begin{tabular}{|l|l|l|l|l|}
\hline \multicolumn{5}{|c|}{ Narrativas empresariales versus estrategias de diseño } \\
\hline & $\begin{array}{l}\text { Narrativa de } \\
\text { explotación }\end{array}$ & Tecno-narrativa & $\begin{array}{l}\text { Narrativa } \\
\text { centrado en el } \\
\text { usuario }\end{array}$ & $\begin{array}{l}\text { Narrativa } \\
\text { explorativa }\end{array}$ \\
\hline $\begin{array}{l}\text { Orientación del } \\
\text { Diseño. }\end{array}$ & $\begin{array}{l}\text { Reconfiguración } \\
\text { de estilos }\end{array}$ & $\begin{array}{l}\text { Búsqueda de } \\
\text { nuevas } \\
\text { aplicaciones }\end{array}$ & $\begin{array}{l}\text { 1.1.1. Explotación } \\
\text { de tecnología en } \\
\text { nuevos mercados }\end{array}$ & $\begin{array}{l}\text { 1.1.2. Innovación modelo del } \\
\text { mistema/negocios. } \\
\text { sel }\end{array}$ \\
\hline $\begin{array}{l}\text { Fuentes de } \\
\text { creatividad }\end{array}$ & $\begin{array}{l}\text { Tendencias extra- } \\
\text { sectoriales } \\
\text { /estímulo }\end{array}$ & 1.1.3. Futurología & $\begin{array}{l}1.1 .5 . \text { Evolución } \\
\text { de signos } \\
\text { relacionados a } \\
\text { productos, } \\
\text { espacios } \\
\text { contenidos y }\end{array}$ & $\begin{array}{l}\text { 1.1.6. Perspectivas } \\
\text { partes interesadas } \\
\text { cognitivos de loma de las } \\
\text { usuarios }\end{array}$ \\
\hline
\end{tabular}

Fuente : DesignIssues: Volume 30, Number 1 Winter (2014)

\footnotetext{
${ }^{32}$ Narrativa, se considera una parcela de eventos secuenciales e interconectados con un comienzo, una conclusión y una estructura básica ( Fisher, 1935, pág. 74)
} 
Valor de Marca, David Aaker ${ }^{33}$, se ha destacado en el mundo del Branding por estudiar y analizar en profundidad la marca y todos los aspectos relacionados con ella, desde la óptica disciplinar del marketing. Aaker considera "el valor de marca como una combinación de conocimiento, lealtad y asociaciones de marca, que se suman para proporcionar valor a un producto o servicio" (Aaker D. , 1996). El autor afirma que el primer paso que hay dar, para iniciar la gestión de la marca, es desarrollar una identidad de marca, que es un conjunto único de asociaciones que se vinculan a lo que la marca pretende representar. La identidad de marca, para Aaker según su modelo es un conjunto de 12 elementos que caen bajo cuatro perspectivas: Marca como producto, Marca como organización, Marca como persona y Marca como símbolo. Su aportación genera alteración en el campo investigativo, lo que da inicio a numerales estudios relacionados, es así que su hija Jennifer Aaker identifica que para construir una identidad hay que considerar tres dimensiones: "la Personalidad que construye la marca al comunicar, el discurso de todo lo que tiene que decir y las expresiones que deberá utilizar para que puedan ser captados" (Aaker J., 1997).

La personalidad de marca se constituye en un componente intangible de la marca, y es definido por su autora "como el conjunto de características humanas asociadas a una marca" (Aaker J. , 1997), bajo este contexto se crea un modelo que permite medir la personalidad de la marca. En el proceso de creación se utilizó 37 marcas en diversas categorías, lo que le permitió detectar 114 rasgos que las describen. El resultado final de este estudio desarrollado en Estados Unidos, presenta un instrumento para medir la personalidad de marca que consta de quince rasgos y cinco dimensiones: sinceridad (práctico, honesto, sano, alegre); emocionante (atrevido, animado, imaginativo, actualizado); competencia (confiable, inteligente, exitoso); sofisticado (clase superior, encantador); rudeza (abierto, resistente). La personalidad de la marca es un componente indispensable a ser considerado en el proceso de diseño, si se visualiza este proceso desde una óptica sistémica, se da la fase de análisis, en la que se debe llegar a la conceptualización del tangible resultante, el mismo que debe tener impregnado la esencia de la marca empresarial.

\section{Conclusiones}

Como protagonistas de la sociedad del conocimiento, se puede apreciar la evolución de las dinámicas sociales, empresariales y culturales que surgen ante la inminente influencia de las innovaciones tecnológicas. El área de diseño no está alejado de esta influencia, muy por el contrario el diseño ha sido movido progresivamente desde los objetos tangibles (donde su enfoque central era su valor de uso), hacia los aspectos vinculados a la intangibilidad: la experiencia de compra, la dinámica de acceso del producto, su disponibilidad y su nivel de nnovación, los que a más del uso, se han constituido en los elementos diferenciadores que crean valor para los usuarios. La integración entre los componentes tangibles e intangibles se ha convertido en un área importante de estudio para él diseño.

Al realizar un recorrido bibliográfico sobre la inclusión de los intangibles empresariales en el proceso de diseño, se pudo evidenciar que es realmente escasa la información, la mayoría de los resultados se concentran en estudiar necesidades, preferencias e intereses del consumidor, reconocidas como variables externas a la empresa, sin considerar las variables internas originadas por los intangibles empresariales. Se ha podido identificar un total de 9 intangibles empresariales, que pueden ser medidos y registrados en los estados financieros por el área contable.

La integración de estos componentes intangibles en el proceso de diseño bajo una visión sistémica, hace necesario que el diseñador adquiera nuevas destrezas. En el rol de intérprete de las narrativas empresariales requerirá necesariamente conocer términos y conceptos administrativos. En el rol de interlocutor de la cultura organizacional, conformada por valores, normas y creencias, visualizada como personalidad de marca, deberá tener nociones de psicología. El desarrollo de la capacidad de Innovación, un intangible de gran potencial en la empresa, pone al diseñador en otro rol, debe ser un futurólogo, que explote al máximo su capacidad creativa. La sostenibilidad del planeta, exigirá que el diseñador comprenda su compromiso por el medio ambiente, por lo que deberá tener nociones de esto también. Hay más variables a considerar dentro del proceso y mucho que investigar aún, este análisis es solo un

\footnotetext{
${ }^{33}$ David Aaker, es profesor emérito de ET Grether de Estrategia de Marketing de la Escuela de Negocios Haas. Es consultor de marketing y autor de más de 100 artículos y unos 15 libros sobre marca. Es considerado uno de los líderes mundiales en su especialidad asesorando empresas de Estados Unidos, Japón y Europa. Es Vicepresidente de la consultora Prophet Management Team.
} 
pequeño aporte al estado del arte de los intangibles empresariales en la sociedad del conocimiento y su implicación en el proceso del diseño.

\section{Bibliografía}

AAKER , D. (1996). Building Strong Brands. New York: The free press.

AAKER, J. (1997). Dimensions of brand personality. JMR, Journal of Marketing Research., 34.

AGUILÁ, J., \& MONGUET, J. (2010). Por que algunas empresas tienen exito y otras no. Barcelona: Grupo Planeta.

ANARGYROS, S., \& LOEB, F. (1998). Qué pasa si ponemos los relojes de nuevo a cero? Hipótesis para futuros probables. Paris: Les Editions de l'imprimeur.

BELL, D. (1991). El advenimiento de la sociedad post-industrial. Madrid: Alianza .

CAÑIBANO, L., \& GISBERT , A. (2005). Principales cambios en la valoración de intangibles. Madrid: AECA, .

CASTELLS, M. (1999). La era de la información. Economía, sociedad y cultura. Madrid: Alianza editorial.

CORRADO, C., HULTEN, C., \& SICHEL, D. (2004). Measuring capital and technology : An expanded framework". Federal Reserve Board, Washington, D.C.

CZARNIAWSKA, B. (1997). Narrating the Organization. Dramas of Institutional Identity . Chicago: The University of Chicago Press.

DESERTI, A., \& RIZZO, F. (2014). Design and the Cultures. DesignIssues, 36-56.

DRUCKER, P. (1969). La era de la discontinuidad.

DRUCKER, P. (2000). El management del siglo XXI. Edhasa.

EUROPEAN COMMISSION. (2003). Study on the measurement of intangible assets and associated reporting practices.

GINER DE LA FUENTE, F., \& GIL ESTALLO, M. (2014). La organización de empresas : Hacia un modelo de futuro. Madrid: ESIC.

HERNANDIS, B. (2010). Metodología del Diseño. Valencia: Departamento de Ingenería gráfica.

MARX, K. (1844). Los Manuscritos económico-filosóficos.

MOOTEE, I. (2014). Design Thinking para la innovacion estrategica. Barcelona: Urano S.A.

MORAN, J., \& BRIGTHTMAN, B. (2001). Leading Organizational Change. Carrer Development International, 111-18.

NORMAN, D., \& VERGANTI, R. (2014). Incremental and radical Innovation : Design rearch vs technology and Meaning Change. DesignIssues, 79.

TORRES, L. (2014). La importancia de los activos intangibles en la sociedad del conocimiento . uexternado, 1-35.

ZURLO, F., \& CAUTELA, C. (2014). Design Strategies in Different. DesignIssues, 19-35. 